Orthopäde $2012 \cdot 41: 93$

DOI 10.1007/s00132-011-1859-6

Online publiziert: 18. Februar 2012

(c) Springer-Verlag 2012
S. Rupp ${ }^{1,2}$

${ }^{1}$ Fachklinik für Orthopädie und Rheumatologie, MediClin Bliestal Kliniken, Blieskastel

${ }^{2}$ Spezielle Orthopädische Chirurgie, Kreiskrankenhaus St. Ingbert

\title{
Aktuelle Aspekte in der konservativen Orthopädie
}

Sehr geehrte Leserinnen und Leser, traditionell ist das Fach Orthopädie gleichwertig konservativ und chirurgisch ausgerichtet.

Durch die Zusammenführung des Fachs mit der Unfallchirurgie überwiegen in der Praxis der Weiterbildung deutlich chirurgische Inhalte. Dabei gewinne ich sicherlich sehr subjektiv - den Eindruck, dass sich mancherorts der Blickwinkel zu stark auf „das Chirurgische“ verengt.

Ist diese Entwicklung wirklich im Interesse der Ärzte und der Patienten?

Ich zweifle daran und sehe darin zumindest die Mitursache für Probleme, die uns teilweise unter scheinbar anderen Vorzeichen beschäftigen:

- der Vorhalt, in Deutschland werde zu viel operiert,

- der relativ hohe Anteil unzufriedener Patienten z. B. nach Wirbelsäuleneingriffen und Knieendoprothesenimplantationen,

- das Ausweichen von Patienten in den nichtärztlichen Versorgungsbereich (Heilpraktiker, nichtärztliche Osteopathen, Chiropraktiker etc.).

Es scheint zumindest so zu sein, dass wir für einen Teil der Patienten, die unsere Hilfe suchen, keine adäquaten medizinischen Lösungen anzubieten haben.

Auf der Suche nach solchen Lösungen lohnt die kritische Auseinandersetzung mit Therapiekonzepten wie Injektionsbehandlung, Triggerpunktbehandlung, Akupunktur, Osteopathie ohne „Patentlösungsanspruch“.

Die Psyche ist weder mit Injektionen noch Inzisionen therapierbar. Die Psyche ist aber immer mit im Spiel. Wir sollten uns von der dichotomen Betrachtung des ,entweder oder" lösen und das ,und“ im Sinne von „mehr oder weniger" akzeptieren. Die Psychosomatik verbindet bereits begrifflich Psyche und Körper. Es ist sinnvoll, diese Erkenntnisse in Indikationsstellungen und auch Ergebnisbewertungen einzubeziehen. Die Anschlussheilbehandlung verbindet exemplarisch spezielle orthopädische Chirurgie und konservative Orthopädie. Am Beispiel der „Reha nach Knie-TEP“ sollen die Möglichkeiten konservativer Orthopädie dargestellt werden. Die Konzepte orientieren sich an einem umfassenderen Krankheitsbegriff (ICF der WHO) und an trainingswissenschaftlichen Grundlagen.

Aus meiner Sicht verdient die konservative Orthopädie einen höheren Stellenwert. Ich glaube nicht, dass es in unserem Interesse sein kann, das Feld anderen, z. B. nichtärztlichen Therapeuten, zu überlassen. Nur der fachkundige Blick ,auf das Ganze“ und damit die fachärztliche Kompetenz gewährleistet die hohe Behandlungsqualität, der wir uns verpflichtet fühlen.

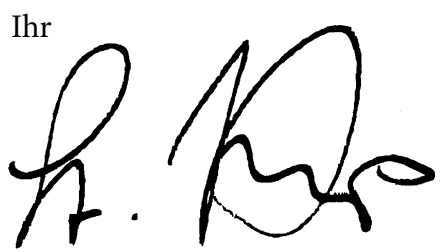

Prof. Dr. S. Rupp

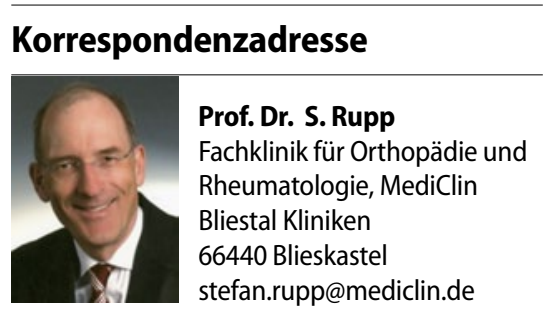

Interessenkonflikt. Der korrespondierende Autor gibt an, dass kein Interessenkonflikt besteht. 